\title{
GABA promotes survival and axonal regeneration in identifiable descending neurons after spinal cord injury in larval lampreys
}

Daniel Romaus-Sanjurjo ${ }^{1}$, Rocío Ledo-García', Blanca Fernández-López,3, Kendra Hanslik², Jennifer R. Morgan², Antón Barreiro-Iglesias (1) ${ }^{1}$ and María Celina Rodicio ${ }^{1}$

\begin{abstract}
The poor regenerative capacity of descending neurons is one of the main causes of the lack of recovery after spinal cord injury (SCl). Thus, it is of crucial importance to find ways to promote axonal regeneration. In addition, the prevention of retrograde degeneration leading to the atrophy/death of descending neurons is an obvious prerequisite to activate axonal regeneration. Lampreys show an amazing regenerative capacity after SCl. Recent histological work in lampreys suggested that GABA, which is massively released after a $\mathrm{SCl}$, could promote the survival of descending neurons. Here, we aimed to study if GABA, acting through GABAB receptors, promotes the survival and axonal regeneration of descending neurons of larval sea lampreys after a complete $\mathrm{SCl}$. First, we used in situ hybridization to confirm that identifiable descending neurons of late-stage larvae express the gabab1 subunit of the GABAB receptor. We also observed an acute increase in the expression of this subunit in descending neurons after $\mathrm{SCl}$, which further supported the possible role of GABA and GABAB receptors in promoting the survival and regeneration of these neurons. So, we performed gain and loss of function experiments to confirm this hypothesis. Treatments with GABA and baclofen (GABAB agonist) significantly reduced caspase activation in descending neurons 2 weeks after a complete SCl. Long-term treatments with GABOB (a GABA analogue) and baclofen significantly promoted axonal regeneration of descending neurons after $\mathrm{SCl}$. These data indicate that GABAergic signalling through GABAB receptors promotes the survival and regeneration of descending neurons after SCl. Finally, we used morpholinos against the gabab1 subunit to knockdown the expression of the GABAB receptor in descending neurons. Long-term morpholino treatments caused a significant inhibition of axonal regeneration. This shows that endogenous GABA promotes axonal regeneration after a complete $\mathrm{SCl}$ in lampreys by activating GABAB receptors.
\end{abstract}

Correspondence: Antón. Barreiro-lglesias (anton.barreiro@usc.es) or María Celina Rodicio (mcelina.rodicio@usc.es)

'Department of Functional Biology, CIBUS, Faculty of Biology, Universidade de Santiago de Compostela, 15782 Santiago de Compostela, Spain

${ }^{2}$ Eugene Bell Center for Regenerative Biology and Tissue Engineering; Marine Biological Laboratory, 7 MBL St., Woods Hole, MA 02543, USA

Full list of author information is available at the end of the article.

These authors contributed equally: Antón Barreiro-Iglesias, María Celina Rodicio

Edited by: A. Verkhratsky

\section{Introduction}

In contrast to mammals, lampreys show spontaneous and successful functional recovery after a complete spinal cord injury (SCI) and this is in part due to their impressive ability for axonal regeneration ${ }^{1-8}$. But, even in lampreys, not all descending neurons of the brain are able to regenerate their axons through the site of injury after a complete spinal cord transection ${ }^{4,9-12}$. The lamprey

\section{(c) The Author(s) 2018}

(c) (i) Open Access This article is licensed under a Creative Commons Attribution 4.0 International License, which permits use, sharing, adaptation, distribution and reproduction cc) in any medium or format, as long as you give appropriate credit to the original author(s) and the source, provide a link to the Creative Commons license, and indicate if changes were made. The images or other third party material in this article are included in the article's Creative Commons license, unless indicated otherwise in a credit line to the material. If material is not included in the article's Creative Commons license and your intended use is not permitted by statutory regulation or exceeds the permitted use, you will need to obtain permission directly from the copyright holder. To view a copy of this license, visit http://creativecommons.org/licenses/by/4.0/. 
brainstem contains approximately 30 large individually identifiable descending reticulospinal neurons that vary greatly in their ability for axonal regeneration after SCI, even when their axons run in similar paths in a spinal cord that is permissive for axonal regrowth ${ }^{4,12,13}$. Some identifiable descending neurons of lampreys are considered "good regenerators" (i.e. they regenerate their axon more than $55 \%$ of the times; the I3, I4, I5, B2, B5 and B6 neurons) and others are considered "bad regenerators" (i.e. they regenerate their axon less than $50 \%$ of the times; the M1, M2, M3, I1, I2, B1, B3, B4 and Mth neurons) ${ }^{4,6,12}$. This indicates that interactions with the extrinsic spinal cord environment and intrinsic differences between descending neurons affect their regenerative abilities after SCI. Recent work has also shown that identifiable descending neurons of lampreys that are known to be "bad regenerators" slowly die after a complete SCI and are also "poor survivors"12,14,15. The death of these neurons after SCI appears to be apoptotic as indicated by the appearance of TUNEL labelling and activated caspases in their soma $^{14-18}$. This offers a convenient vertebrate model to study the inhibition or promotion of neuronal survival and axonal regeneration in the same in vivo preparation and at the level of single neurons.

In mammals, SCI leads to a massive release of aminoacidergic neurotransmitters (glycine and GABA: ${ }^{19,20}$; glutamate: ${ }^{21-23}$ ). Excessive glutamate release after SCI is responsible for excitotoxicity and neuronal death ${ }^{21,22}$. High extracellular glutamate levels result in excessive activation of glutamate receptors, triggering massive $\mathrm{Ca}^{2+}$ influx into cells, which leads to neuronal death ${ }^{24}$. Extracellular glycine could also contribute to glutamate excitotoxicity ${ }^{20}$, since it is a co-agonist of the $\mathrm{N}$-methyl-D-aspartate glutamate receptor ${ }^{25}$. The phenomenon of excitotoxicity has been mainly studied in intrinsic spinal cord cells; however, retrograde damage to neurons is also likely due to the fact that $\mathrm{Ca}^{2+}$ ions gain access to the axoplasm of damaged axons ${ }^{26}$. In contrast to glutamate, it has been reported that GABA could have neuroprotective effects after different types of central nervous system (CNS) damage $\mathrm{e}^{27-31}$. The activation of pre-synaptic GABAB receptors causes inactivation of voltage-dependent $\mathrm{Ca}^{2+}$ channels $\left(\mathrm{see}^{32}\right.$ ), which could prevent the influx of $\mathrm{Ca}^{2+}$ ions due to glutamate release. In addition, it has been shown that GABA can modulate and promote neurite outgrowth in vitro or during development (for reviews $\mathrm{see}^{33,34}$ ). However, a role for GABA and GABAB receptors in neuroprotection and especially in axonal regeneration after SCI has not been reported yet.

In lampreys, glutamate induces an inhibition of neurite outgrowth in reticulospinal neurons in vitro due to $\mathrm{Ca}^{2+}$ influx $^{35}$. Electrophysiological studies have also suggested that low intracellular $\mathrm{Ca}^{2+}$ levels due to downregulation of $\mathrm{Ca}^{2+}$ channels could facilitate axonal regeneration in axotomized descending neurons of lampreys ${ }^{36}$. More recently, we have reported that, as in mammals, there is a massive release of glutamate, GABA and glycine from most spinal cord neurons close to the lesion site following a complete $\mathrm{SCI}^{37-39}$. Between 1 and 3 days after the injury, we observed the extracellular accumulation of GABA in the form of "halos" around some axotomized axons of descending neurons close to the site of injury. Statistical analyses revealed a significant correlation between GABA accumulation and a higher survival ability of the corresponding identifiable descending neurons ${ }^{37}$. An electrophysiological study in the spinal cord of lampreys has also found a correlation between higher GABAergic inhibition and a better recovery of function in spinal lesioned animals ${ }^{40}$. These data prompted us to hypothesize that, in lampreys, increased GABA signalling after SCI could be favouring the recovery process by promoting survival and axonal regeneration of descending neurons. Here, we address this question for the first time in vivo in any vertebrate and provide gain and loss of function evidence showing that endogenous GABA, acting through GABAB receptors, promotes survival and axonal regeneration of identifiable descending neurons after SCI in lampreys.

\section{Materials and methods \\ Animals}

All experiments involving animals were approved by the Bioethics Committee at the University of Santiago de Compostela and the Consellería do Medio Rural e do Mar of the Xunta de Galicia (License reference JLPV/IId; Galicia, Spain) or the Institutional Animal Care and Use Committee at the Marine Biological Laboratory (Woods Hole, MA) and were performed in accordance to European Union and Spanish guidelines on animal care and experimentation or the National Institutes of Health, respectively. During experimental procedures, special effort was taken to minimize animal suffering and to reduce the use of animals. Animals were deeply anaesthetized with $0.1 \%$ MS-222 (Sigma, St. Louis, MO) in lamprey Ringer solution before all experimental procedures and euthanized by decapitation at the end of the experiments.

Mature and developmentally stable larval sea lampreys, Petromyzon marinus L. ( $n=115$; between 95 and $120 \mathrm{~mm}$ in body length, 5 to 7 years of age), were used in the study. Larval lampreys were collected from the river Ulla (Galicia, Spain), with permission from the Xunta de Galicia, or provided by Lamprey Services, Inc. (Ludington, MI, USA) and maintained in aerated fresh water aquaria at $15-23^{\circ} \mathrm{C}$ with a bed of river sediment until their use in experimental procedures. Lampreys were randomly distributed between the different experimental groups.

\section{$\mathrm{SCl}$ surgical procedures}

Animals were assigned to the following experimental groups: control unlesioned animals or animals with a 
Table 1 Table showing the number of animals included in each experimental group and also the total number of identifiable descending neurons that were included in the analyses

\begin{tabular}{|c|c|c|c|c|c|c|c|c|c|c|c|c|c|c|c|c|c|}
\hline & & \multirow[t]{2}{*}{ Animals } & \multicolumn{15}{|c|}{ Total number of neurons included in the analyses } \\
\hline & & & M1 & M2 & M3 & 11 & 12 & 13 & 14 & 15 & B1 & B2 & B3 & B4 & B5 & B6 & Mth \\
\hline \multirow[t]{3}{*}{ Changes in gabab1 expression } & Control & 7 & 11 & 11 & 12 & 8 & - & 5 & 8 & 5 & 9 & - & 10 & 9 & - & 7 & 9 \\
\hline & $1 \mathrm{wpl}$ & 7 & 10 & 10 & 14 & 12 & - & 12 & 9 & 6 & 9 & - & 13 & 9 & - & 11 & 14 \\
\hline & 4 wpl & 6 & 10 & 12 & 12 & 9 & - & 6 & 6 & 6 & 5 & - & 6 & 5 & - & 5 & 7 \\
\hline \multirow[t]{2}{*}{ GABA treatment (2 wpl) } & Control & $6^{*}$ & 11 & 11 & 10 & 12 & 9 & 11 & 12 & 7 & 12 & 11 & 12 & 12 & 11 & 12 & 12 \\
\hline & Treated & 5 & 6 & 8 & 8 & 9 & 6 & 9 & 9 & 5 & 10 & 10 & 10 & 10 & 7 & 9 & 10 \\
\hline \multirow[t]{2}{*}{ Baclofen treatment (caspase activation, 2 wpl) } & Control & $7^{*}$ & 13 & 12 & 11 & 14 & 9 & 13 & 14 & 8 & 14 & 13 & 14 & 14 & 12 & 14 & 14 \\
\hline & Treated & 7 & 12 & 13 & 13 & 14 & 7 & 14 & 13 & 6 & 14 & 13 & 14 & 14 & 13 & 13 & 13 \\
\hline \multirow[t]{2}{*}{ GABOB treatment (12 wpl) } & Control & 15 & 30 & 30 & 29 & 29 & 24 & 30 & 30 & 29 & 30 & 30 & 30 & 30 & 30 & 30 & 30 \\
\hline & Treated & 14 & 26 & 25 & 24 & 28 & 24 & 28 & 27 & 28 & 25 & 24 & 26 & 28 & 23 & 28 & 24 \\
\hline \multirow[t]{2}{*}{ Baclofen treatment (axonal regeneration, 12 wpl) } & Control & 7 & 12 & 12 & 13 & 14 & 12 & 14 & 14 & 11 & 14 & 12 & 14 & 14 & 14 & 12 & 13 \\
\hline & Treated & 11 & 21 & 21 & 21 & 22 & 19 & 22 & 22 & 21 & 22 & 22 & 22 & 22 & 22 & 22 & 22 \\
\hline \multirow[t]{2}{*}{ Gabab1 morpholino (ISH, 2 wpl) } & Control & 3 & 6 & - & - & 6 & - & - & - & - & - & - & - & - & - & - & - \\
\hline & Treated & 4 & 7 & - & - & 8 & - & - & - & - & - & - & - & - & - & - & - \\
\hline \multirow[t]{3}{*}{ Gabab1 morpholino (axonal regeneration, 10 wpl) } & Control & 9 & 18 & 18 & 18 & 18 & 18 & 18 & 18 & 18 & 18 & 18 & 18 & 18 & 18 & 18 & 18 \\
\hline & Treated & 13 & 26 & 26 & 26 & 25 & 26 & 26 & 26 & 26 & 26 & 26 & 26 & 26 & 26 & 26 & 26 \\
\hline & Total & 115 & & & & & & & & & & & & & & & \\
\hline
\end{tabular}

Please note that in the in situ hybridization experiments, only the neurons that were unequivocally identified in at least two brain sections were included in the quantifications. In the FLICA experiments, six animals were used as controls for both the GABA and baclofen treatments and an extra animal was used as a control only for the baclofen treatment (asterisks)

complete spinal cord transection that were analyzed 1 week post-lesion (wpl), $2 \mathrm{wpl}, 4 \mathrm{wpl}, 10 \mathrm{wpl}$ or $12 \mathrm{wpl}$. Within the 2, 10 and $12 \mathrm{wpl}$ groups, the injured animals were assigned to either control or treatment groups. Table 1 summarizes the number of animals assigned to each experimental group and condition. Each experiment was carried out in at least two different batches of animals. Complete spinal cord transections were performed as previously described ${ }^{41}$. Briefly, the rostral spinal cord was exposed from the dorsal midline at the level of the 5th gill by making a longitudinal incision with a scalpel (\#11). A complete spinal cord transection was performed with Castroviejo scissors and the spinal cord cut ends were visualized under the stereomicroscope. After spinal transections, the animals were returned to fresh water tanks and each transected animal was examined $24 \mathrm{~h}$ after surgery to confirm that there was no movement caudal to the lesion site. Then, the animals were allowed to recover in individual fresh water tanks at $19.5^{\circ} \mathrm{C}$ and in the dark.

\section{In situ hybridization}

For gabab1 in situ hybridization, the head of the animals was fixed by immersion in $4 \%$ paraformaldehyde (PFA) in $0.05 \mathrm{M}$ Tris-buffered saline (TBS; $\mathrm{pH} 7.4$ ) for $12 \mathrm{~h}$. Then, the brains were dissected out, washed and embedded in Neg $50^{\mathrm{TM}}$ (Microm International $\mathrm{GmbH}$, Walldorf, Germany), frozen in liquid nitrogen-cooled isopentane, sectioned on a cryostat in the transverse plane $(14 \mu \mathrm{m}$ thick) and mounted on Superfrost Plus glass slides (Menzel, Braunschweig, Germany). In situ hybridization with a specific riboprobe for the gabab1 subunit of the sea lamprey gabab receptor (GenBank accession number KX655780; see Suppl. Figure 1) was conducted as previously described ${ }^{42}$. Briefly, brain sections were incubated with the sea lamprey gabab1 DIG-labelled probe at $70{ }^{\circ} \mathrm{C}$ and treated with RNAse A (Invitrogen, Massachusetts, USA) in the posthybridization washes. Then, the sections were incubated with a sheep anti-DIG antibody conjugated to alkaline phosphatase (1:2000; Roche, Mannhein, Germany) overnight. Staining was conducted in BM Purple (Roche) at $37^{\circ}$ C. In situ hybridization experiments were performed in parallel with animals from the different experimental groups (control, $1 \mathrm{wpl}, 2 \mathrm{wpl}$ and $4 \mathrm{wpl}$ ) and the colorimetric reaction was stopped simultaneously for all sections from the different groups of animals.

\section{Drug treatments}

The following drugs were used to treat the animals following the complete spinal cord transection: GABA 
(Sigma; Cat\#: A2129; MW: $103.12 \mathrm{~g} / \mathrm{mol}$ ), GABOB (a GABA analogue; Sigma; Cat\#: A56655; MW: $119.12 \mathrm{~g} /$ $\mathrm{mol}$ ) and baclofen (a GABAB receptor agonist). Baclofen was acquired from two different companies: for the experiments of caspase activation, we used baclofen from Molekula (Newcastle, UK; Cat\#: 31184509; MW: 213.66 $\mathrm{g} / \mathrm{mol}$ ), and for the experiments of axonal regeneration, we used baclofen from Carbosynth (Berkshire, UK; Cat\#: FB18127; MW: $213.66 \mathrm{~g} / \mathrm{mol})$. The drugs were applied in the water where the animals were left after the SCI surgical procedures (GABA at a concentration of $500 \mu \mathrm{M}$, GABOB at a concentration of $50 \mu \mathrm{M}$ and baclofen at a concentration of $125 \mu \mathrm{M})$. The concentrations of baclofen and GABA were selected based on previous in vitro electrophysiological studies in lampreys ${ }^{43}$. Since GABA does not easily cross the blood-brain barrier, it was applied at a high concentration and only in the first days after the injury when the spinal cord is still disrupted. We assumed that GABOB and baclofen also cross the blood-brain barrier as in mammals, since the blood-brain barrier of lampreys is similar to that of higher vertebrates ${ }^{44,45}$. While we do not know the final concentration of the drugs in the CNS, we were confident that this application route allows access to the CNS as there were changes in the swimming behaviour of unlesioned animals in pilot experiments using baclofen and GABOB at these concentrations (not shown). Since these drugs are water soluble, control lesioned and non-treated animals were left in fresh water only. The animals that were analyzed for caspase activation $2 \mathrm{wpl}$ were treated with GABA or baclofen during 4 days from the day of injury and replacing the drug and water every day during those 4 days. The animals that were analyzed for axonal regeneration $12 \mathrm{wpl}$ were treated with GABOB or baclofen during the 12 weeks replacing the drug and the water four times each week. The animals were always kept in the dark during the drug treatments to prevent light degradation of these drugs.

\section{Morpholino treatment}

Application of morpholinos was performed as previously described in ref. $^{13}$. Briefly, the spinal cord was transected at the level of the 5th gill (see surgical procedures), and morpholinos $(20 \mu \mathrm{g}$ in lamprey internal solution: $180 \mathrm{mM} \mathrm{KCl}, 10 \mathrm{mM}$ HEPES, $\mathrm{pH}$ 7.4; designed by GeneTools, LLC; Philomath, OR) were added at the time and site of SCI soaked in a small piece of Gelfoam (Pfizer; New York, NY). These included an active splicingblocking gabab1 morpholino (5'-ACGTCTGCAACGGAGAGTCATGAGA-3') generated against the boundary between the second intron and the second exon of the partial sea lamprey gabab1 sequence (Suppl. Figure 1), and a 5-base pair mismatch gabab1 negative control morpholino (5'-ACcTCTcCAACcGAGAcTCATcAGA- $\left.3^{\prime}\right)$. During recovery, the morpholinos are retrogradely transported to the cell soma of descending neurons where they can knockdown the expression of the target mRNA $^{13,46-48}$. Animals were allowed to recover for 10 wpl to analyze the effect of gabab1 knockdown (KD) in axonal regeneration of identifiable descending neurons. In situ hybridization was used to control the efficacy of the gabab1 morpholino KD in animals processed at $2 \mathrm{wpl}$.

\section{Detection of activated caspases in whole-mounted brain preparations}

The Image-iT LIVE Green Poly Caspases Detection Kit (Cat. No. I35104, Invitrogen, USA) was used to detect activated caspases in identifiable descending neurons (the M1, M2, M3, I1, I2, I3, I4, I5, B1, B2, B3, B4, B5, B6 and Mth neurons; see Suppl. Figure 2A) of larval sea lampreys 2 weeks after the complete spinal cord transection and the GABA or baclofen treatments. This kit contains 1 vial (component A of the kit) of the lyophilized FLICA reagent (FAM-VAD-FMK). The reagent associates a fluoromethyl ketone (FMK) moiety, which can react covalently with a cysteine, with a caspase-specific aminoacid sequence (valine-alanine-aspartic acid (VAD)). A carboxyfluorescein group (FAM) is attached as a fluorescent reporter. The FLICA reagent interacts with the enzyme active centre of an activated caspase via the recognition sequence, and then attaches covalently through the FMK moiety. Experiments for the detection of activated caspases in whole-mounted brain preparations were done as previously described ${ }^{16-18}$. Briefly, brains from control and treated $2 \mathrm{wpl}$ animals were dissected out and immediately incubated in $150 \mu \mathrm{L}$ of phosphate buffered saline (PBS) containing $1 \mu \mathrm{L}$ of the $150 \times$ FLICA labelling solution at $37^{\circ} \mathrm{C}$ for $1 \mathrm{~h}$. Then, the brains were washed with PBS. Brains were fixed in $4 \%$ PFA in PBS for $2 \mathrm{~h}$ and $30 \mathrm{~min}$ at $4{ }^{\circ} \mathrm{C}$. Next, the brains were washed with PBS, mounted on Superfrost Plus glass slides, and mounted with Mowiol.

\section{Retrograde labelling of descending neurons with regenerated axons}

At 10 (morpholino treatments) or 12 (GABOB and baclofen treatments) wpl, a second complete spinal cord transection was performed $5 \mathrm{~mm}$ below the site of the original transection and the retrograde tracer Neurobiotin (NB, 322.8 Da molecular weight; Vector; Burlingame, CA) was applied to the spinal cord lesion with the aid of a Minutien pin (\#000). The animals were allowed to recover at $19.5^{\circ} \mathrm{C}$ with appropriate ventilation conditions for 7 days to allow the transport of the tracer from the application point to the neuronal soma of identifiable descending neurons (the M1, M2, M3, I1, I2, I3, I4, I5, B1, B2, B3, B4, B5, B6 and Mth were analyzed; see Suppl. Figure 2A). Since the original SCI also was a complete spinal cord transection, only neurons whose axons 
regenerated at least $5 \mathrm{~mm}$ below the site of injury were labelled by the tracer. Brains of these larvae were dissected out, and the posterior and cerebrotectal commissures of the brain were cut along the dorsal midline, and the alar plates were deflected laterally and pinned flat to a small strip of Sylgard (Dow Corning Co., USA) and fixed with $4 \%$ PFA in TBS for $4 \mathrm{~h}$ at room temperature. After washes in TBS, the brains were incubated at room temperature with Avidin D-FITC conjugated (Vector; Cat\#: A-2001; 1:500) diluted in TBS containing $0.3 \%$ Triton $X-100$ for 2 days to reveal the presence of Neurobiotin. Brains were rinsed in TBS and distilled water and mounted with Mowiol.

\section{Imaging and quantifications}

An Olympus photomicroscope (AX-70; Provis) with a $20 \times$ Apochromatic 0.75 lens and equipped with a colour digital camera (Olympus DP70, Tokyo, Japan) was used to acquire images of brain sections from the in situ hybridization experiments. Images were always acquired with the same microscope and software settings. For the quantification of the level of gabab1 positive signal in identifiable descending neurons, first we established the intensity rank of positive colorimetric in situ signal. For this, we analyzed 10 random images from different descending neurons of control and lesioned animals. The "histogram" function in Image J shows the number of pixels in each image in a range of intensity from 0 to 255 . With these images, we compared the intensity values in regions with clear in situ signal and the intensity values in regions without in situ signal. Based on this, we established a value of 179 as the lower limit to consider the colorimetric in situ signal as positive. Then the number of pixels of positive in situ signal was quantified for each section of each identified descending neuron. In brain sections, the identification of some of the specific descending cells becomes more difficult than in wholemounts. Thus, only the cells that were unequivocally identified in at least two different sections were included in the quantifications (the M1, M2, M3, I1, I3, I4, I5, B1, B3, B4, B6 and Mth neurons; see Suppl. Figure 2A). Then, we calculated the average number of positive pixels per section for each individual neuron (see Table 1) and these data were used for statistical analyses. The experimenter was blinded during quantifications.

The quantification of the intensity of FLICA labelling was done as previously described ${ }^{18}$. Briefly, photomicrographs were acquired with a spectral confocal microscope (model TCS-SP2; Leica, Wetzlar, Germany). Images were always acquired under the same microscope conditions for control or treated animals. Quantification of mean fluorescent intensity (mean grey value) of each identifiable neuron was done using the Fiji software. In whole-mounted brain preparations, the specific descending neurons are easily identifiable based on their morphology and rostro-caudal and dorso-ventral anatomical location. The experimenter was blinded during quantifications. The data from each individual identifiable neuron (see Table 1) were used for statistical analyses.

The percentage of neurons with regenerated axons (labelled by the Neurobiotin tracer) with respect to the total number of analyzed neurons (see Table 1) was calculated for each type of identifiable descending neuron using an Olympus microscope or a Zeiss AxioImager Z2 microscope. The percentage of neurons with regenerated axons with respect to the total number of analyzed neurons in each animal was also calculated and these data were used for statistical analyses. The experimenter was blinded during quantifications. For the figures, images were taken with the Olympus microscope or the spectral confocal microscope (model TCS-SP2; Leica).

After quantifications, contrast and brightness were minimally adjusted with Adobe Photoshop CS4 or CS6 (Adobe Systems, San José, CA, USA). Figure plates and lettering were generated using Adobe Photoshop CS4 or CS6 (Adobe Systems).

\section{Statistical analyses}

Statistical analysis was carried out using Prism 6 (GraphPad software, La Jolla, CA). Data were presented as mean \pm S.E.M. Normality of the data was determined only when $n$ numbers were higher than 10 by using the D'Agostino-Pearson omnibus test, and the homoscedasticity was determined by the Brown-Forsythe test. The in situ hybridization data that were normally distributed and homoscedastic were analyzed by a one-way ANOVA. Post-hoc Dunnett's multiple comparison tests were used to compare pairs of data. In situ hybridization data that were not normally distributed (or when the $n$ numbers were lower than 10) were analyzed by a Kruskal-Wallis test and post-hoc Dunn's multiple comparisons test. The results of control vs. treatment groups were analyzed by a Student's $t$-test (for normally distributed data) or a Mann-Whitney $U$ test (for nonnormally distributed data). The in situ hybridization data after morpholino application were analyzed by a Mann-Whitney $U$ test. The significance level was set at 0.05 . In the figures, significance values were represented by different number of asterisks: 1 asterisk ( $p$ value between 0.01 and 0.05 ), 2 asterisks ( $p$ value between 0.001 and 0.01$), 3$ asterisks ( $p$ value between 0.0001 and 0.001 ) and 4 asterisks ( $p$ value $<0.0001)$.

\section{Results}

Increased expression of the gabab1 subunit in identifiable descending neurons after $\mathrm{SCl}$

GABAB receptors are obligate heterodimers formed by gabab1 and gabab2 subunits ${ }^{49}$. In previous work, we 
reported the expression of the gabab1 and gabab2 receptor subunits in identifiable descending neurons of adult sea lampreys under normal conditions ${ }^{42}$. Here, we used gabab1 in situ hybridization first to confirm that this receptor is also expressed in identifiable descending neurons of mature larval sea lampreys (Suppl. Figure 2B; Fig. 1a, c, e) and then to quantify changes in its expression after SCI (Fig. 1a-g; Suppl. Figure 3). The M1, M2, M3, I1, I3, I4, I5, B1, B3, B4, B6 and Mth neurons were included in the analyses (see Material and Methods). This revealed a significant increase in the expression of the gabab1 subunit in the M2 (ANOVA, $p$ $=0.0049)$, M3 (ANOVA, $p=0.002)$, I1 (Kruskal-Wallis, $p=0.0009), \quad$ I3 $\quad$ (Kruskal-Wallis, $\quad p=0.0097), \quad$ B1 (Kruskal-Wallis, $p=0.015$ ) and B3 (Kruskal-Wallis, $p$ $=0.0178$ ) neurons (Fig. 1g; Table 2) in $1 \mathrm{wpl}$ animals as compared to control unlesioned animals. Subsequent power calculations (using $80 \%$ power) indicated that the sample sizes were appropriately powered. Although a similar trend was observed for the M1, I4, I5, B4, B6 and Mth neurons in $1 \mathrm{wpl}$ animals as compared to control unlesioned animals, statistical analyses did not reveal significant changes in the expression of the gabab1 subunit in these neurons (Suppl. Figure 3; Table 2). At $4 \mathrm{wpl}$, the expression of the gabab1 subunit was not significantly different to control unlesioned animals in all identifiable descending neurons and returned to control levels (Fig. 1g; Suppl. Figure 3; Table 2). This shows that the complete SCI induced an acute increase in the expression of the gabab1 subunit in descending neurons, which, together with the accumulation of GABA around the axons of identifiable neurons $^{37}$, supports the possible role of endogenous GABA as a neuroprotective and pro-regenerative molecule after SCI in lampreys.

\section{GABA and baclofen treatments inhibit caspase activation} in descending neurons after $\mathrm{SCl}$

To test our hypothesis, we first analyzed the effect of GABA and baclofen (GABAB agonist) treatments in caspase activation in identifiable descending neurons after a complete SCI using FLICA labelling (Fig. 2a-i). As previously shown, in control lesioned animals, there is a statistically significant correlation between the intensity of FLICA labelling and the long-term survival and regenerative abilities of identifiable neurons (not shown; ${ }^{18,50}$ ). At $2 \mathrm{wpl}$, animals treated with GABA or baclofen during 4 days showed a significant inhibition of caspase activation (fluorescence intensity of FLICA labelling) in identifiable descending neurons as compared to control animals (GABA: Student's $t$-test, $p<0.0001$; baclofen: Student's $t$-test, $p<0.0001$; Fig. $2 \mathrm{j}$, k). This suggests that GABA can inhibit apoptosis in descending neurons after SCI by activating GABAB receptors.

\section{GABOB and baclofen long-term treatments promote axonal regeneration in descending neurons after $\mathrm{SCl}$}

Then, we studied the long-term effect of increasing GABAergic signalling in axonal regeneration after a complete SCI. Retrograde neuronal tract-tracing with Neurobiotin showed that a treatment with either GABOB (Fig. 3) or baclofen (Fig. 4) during 12 weeks post-lesion significantly promoted axonal regeneration of identifiable descending neurons after a complete SCI as compared to control animals (GABOB: Student's $t$ test, $p=0.0129$ (Fig. 3h); baclofen: Mann-Whitney $U$ test, $p=0.0004$ (Fig. 4h)). The baclofen used in these experiments (from Carbosynth) was also tested to confirm that it had the same effect in the activation of caspases as the baclofen acquired from Molekula. This baclofen also inhibited caspase activation significantly in identifiable descending neurons as compared to control animals in a different set of experiments (Mann-Whitney $U$ test, $p<0.0001$; not shown). This shows that an increase in GABAergic signalling through GABAB receptors promotes axonal regeneration after a complete SCI.

\section{Endogenous $G A B A$ signalling through $G A B A B$ receptors promotes axonal regeneration after $\mathrm{SCl}$}

To test whether endogenous GABA also promotes regeneration by activating GABAB receptors, we decided to use morpholinos to knockdown the expression of the gabab1 subunit in descending neurons after a complete SCI (Fig. 5). First, we used in situ hybridization to confirm that the active gabab1 morpholino is able to knockdown the expression of the gabab1 mRNA in identifiable neurons of $2 \mathrm{wpl}$ animals (Fig. 5a-e). As an example, we analyzed the M1 (Fig. 5a, b) and the I1 neurons (Fig. 5c, d). The active gabab1 morpholino was able to significantly knockdown the expression of the gabab1 mRNA in identifiable descending neurons as compared to the gabab1 mismatch control morpholino (M1: Mann-Whitney $U$ test, $p=0.0111$; I1: Mann-Whitney $U$ test, $p=0.0057$; Fig. 5e). Then, neuronal tract-tracing showed that the treatment with the active gabab1 morpholino significantly inhibited axonal regeneration of identifiable descending neurons 10 weeks after a complete $\mathrm{SCI}$ as compared to the animals treated with the gabab1 mismatch control morpholino (Mann-Whitney $U$ test, $p$ $=0.0133$; Fig. $5 \mathrm{f}-\mathrm{m}$ ). This confirms that, in lampreys, endogenous GABA promotes axonal regeneration of descending neurons after a complete SCI by activating GABAB receptors.

\section{Discussion}

Here, we have provided gain and loss of function data, using pharmacological and genetic treatments, showing that endogenous GABA signalling through GABAB 


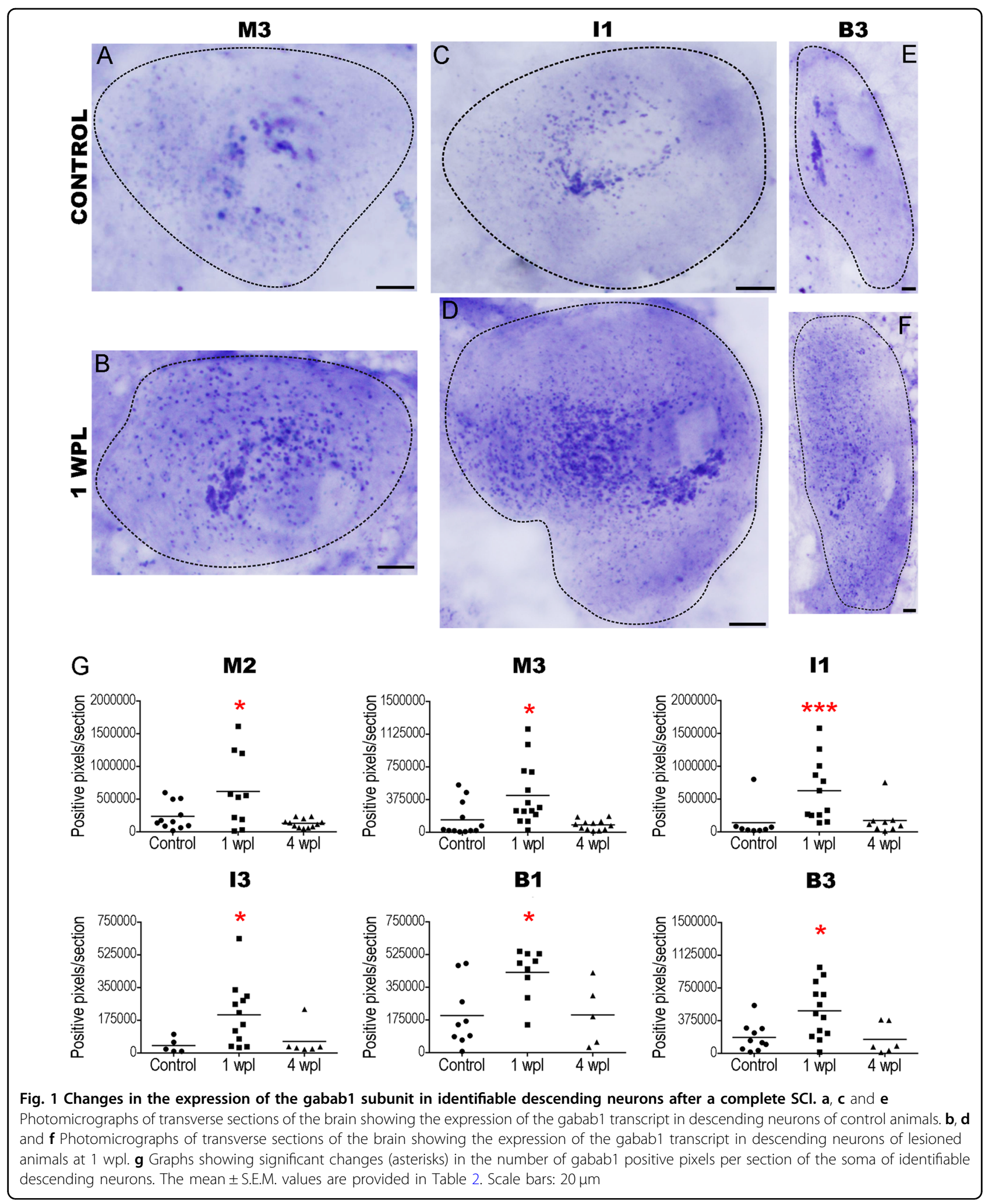

receptors promotes neuronal survival and axonal regeneration of identifiable descending neurons of lampreys after a complete SCI.
The analysis of the changes of expression of the gabab1 subunit in response to a complete $\mathrm{SCI}$ revealed a significant increase in the expression of this subunit in 
Table 2 Mean \pm S.E.M. values of the number of gabab1 positive in situ pixels/section in identifiable descending neurons of control and injured animals

\begin{tabular}{llll}
\hline Gabab1 positive pixels/section & Control & $\mathbf{1}$ wpl & $\mathbf{4}$ wpl \\
\hline M1 & $258,170 \pm 86,006$ & $320,350 \pm 78,104$ & $148,657 \pm 43,920$ \\
M2 & $237,552 \pm 61,602$ & $618,158 \pm 176,397$ & $131,367 \pm 19,089$ \\
M3 & $144,448 \pm 56,162$ & $423,439 \pm 93,059$ & $85,245 \pm 16,702$ \\
I1 & $140,858 \pm 94,768$ & $627,179 \pm 137,246$ & $176,392 \pm 74,810$ \\
I3 & $39,783 \pm 17,599$ & $204,914 \pm 49,099$ & $61,945 \pm 34,492$ \\
I4 & $68,678 \pm 11,645$ & $147,613 \pm 44,903$ & $45,723 \pm 7,610$ \\
I5 & $37,027 \pm 21,463$ & $54,634 \pm 23,587$ \\
B1 & $198,893 \pm 57,286$ & $62,221 \pm 13,380$ & $202,694 \pm 75,156$ \\
B3 & $184,112 \pm 51,392$ & $429,418 \pm 43,503$ & $161,864 \pm 70,596$ \\
B4 & $183,470 \pm 49,454$ & $488,947 \pm 86,189$ & $274,012 \pm 88,414$ \\
B6 & $222,515 \pm 105,034$ & $317,944 \pm 79,980$ & $273,466 \pm 101,442$ \\
Mth & $201,230 \pm 84,794$ & $456,303 \pm 87,734$ & $139,834 \pm 31,750$ \\
\hline
\end{tabular}

Refers to Fig. 1 and Suppl. Figure 2

some identifiable descending neurons (with other neurons showing a similar trend). As stated in the introduction, massive glutamate release and the subsequent activation of glutamate receptors lead to an increase in $\mathrm{Ca}^{2+}$ influx into cells, which causes excitotoxicity and neuronal death after $\mathrm{SCI}^{21,22,24,51}$; $\operatorname{see}^{52}$. GABAB receptors can cause the inactivation of voltage-dependent $\mathrm{Ca}^{2+}$ channels $\left(\mathrm{see}^{32}\right)$. Therefore, this increase in the expression of GABAB receptors could compensate for the influx of $\mathrm{Ca}^{2+}$ into axotomized descending neurons caused by massive glutamate release. The acute increase in the expression of the gabab1 subunit in descending neurons and the massive release of GABA after $\mathrm{SCl}^{37}$ appears as one of the mechanisms favouring neuronal survival and axonal regeneration after SCI in lampreys. As far as we are aware, no study has analyzed the expression of gabab subunits after SCI in mammals. Only a few mammalian studies have looked at changes in the expression of this receptor following other types of nervous system injuries (sciatic nerve ligation: ${ }^{53}$; traumatic brain injury: ${ }^{54}$; ulnar nerve transection: ${ }^{55}$; cerebral ischaemia: ${ }^{56}$ ). In contrast to the present results in lampreys, these studies showed that the expression of GABAB receptors decreases after the injury in different regions of the brain ${ }^{54-56}$. This could be a key difference between regenerating and non-regenerating animals, since axons of the later do not show good regenerative abilities after CNS injuries. Interestingly, and in agreement with the results in lampreys, Huang and colleagues ${ }^{56}$ reported that an elevation in the protein expression of $\mathrm{GABAB}$ receptors in the cerebral cortex promotes neuroprotection after ischaemic damage.
There is some controversy on the topic of whether descending neurons of the brain of mammals die after SCI. Some studies have shown the death of brain neurons after $\mathrm{SCI}^{57-63}$. On the other hand, two recent reports did not find evidence of the death of corticospinal neurons after $\mathrm{SCI}^{64,65}$, and suggested that these neurons only suffer atrophy but do not die ${ }^{65}$. In any case, the death or atrophy of descending neurons of mammals appears to involve apoptotic mechanisms as shown by the appearance of TUNEL labelling and activated caspase-3 immunoreactivity in these neurons after the injury at spinal levels ${ }^{60-}$ ${ }^{62}$. Recent work in lampreys has also shown that identifiable descending neurons known to be "bad regenerators" are actually "poor survivors" after a complete $\mathrm{SCI}^{8,12,14,50}$. These neurons enter in a process of slow and delayed death after $\mathrm{SCI}^{8,12-18}$ that is initiated by caspase activation in the injured axon at spinal levels ${ }^{17,18}$. The death of these neurons also occurs through apoptotic mechanisms as shown by the appearance of activated caspases ${ }^{15-18}$, TUNEL labelling ${ }^{14,15}$ and Fluoro-Jade ${ }^{\circledR} \mathrm{C}$ labelling ${ }^{12,18}$. Recent results have shown that there is a significant correlation between the intensity of caspase activation $2 \mathrm{wpl}$ and the long-term regenerative ${ }^{18}$ and survival ${ }^{50}$ abilities of identifiable descending neurons of lampreys after SCI. Present results indicate that the activation of GABAB receptors by GABA/baclofen can inhibit caspase activation after SCI in identifiable descending neurons, which is a key step to preventing the development of apoptosis and promoting neuronal survival. Previous work in other models of CNS injury also showed that a baclofen treatment can inhibit caspase activation (model of kainic-acidinduced seizure in rats: ${ }^{29}$; models of ischaemic brain injury 


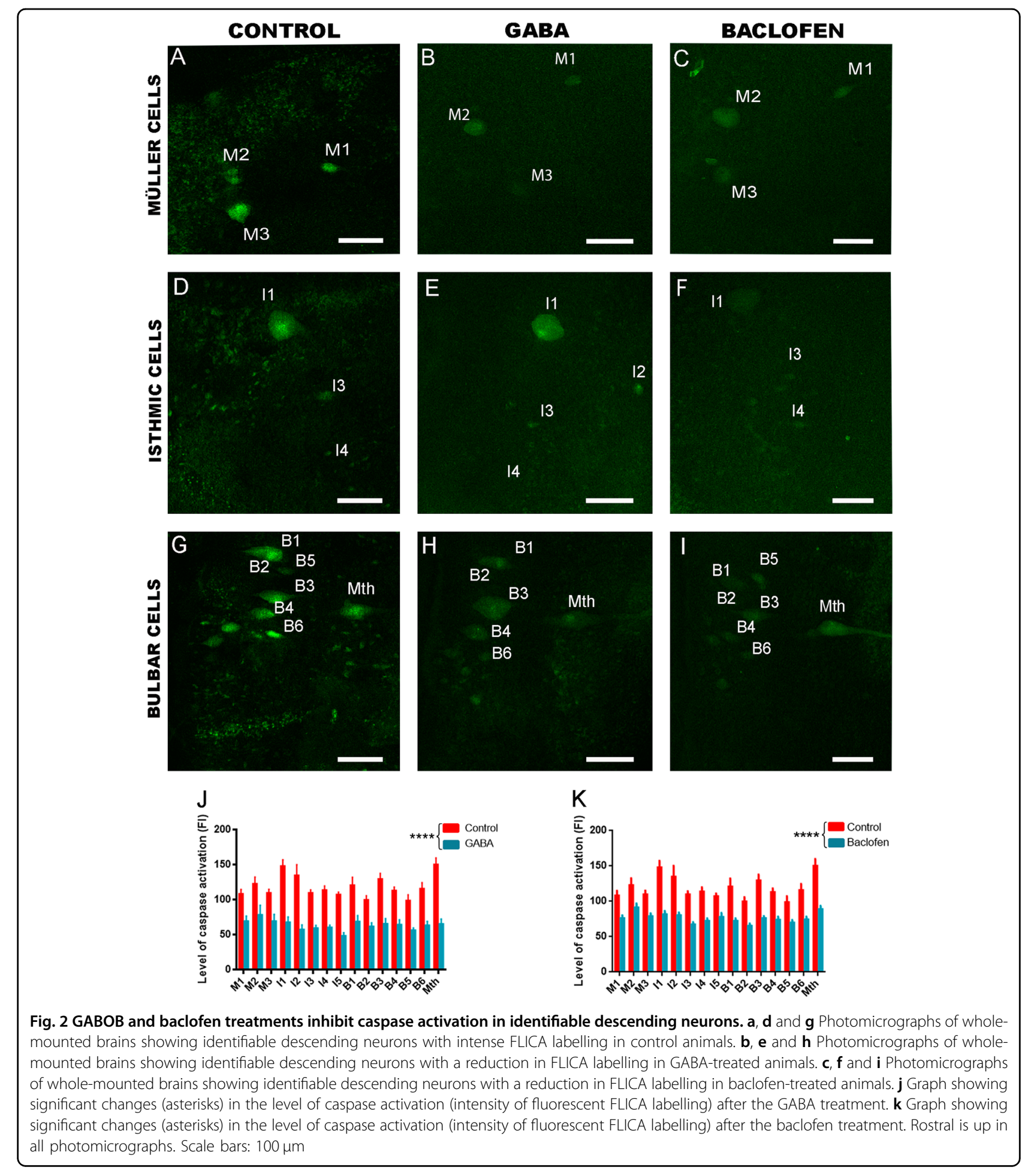

in rats: ${ }^{27,31}$; model of chemical hypoxia in retinal ganglion cells in rats: $\left.{ }^{66}\right)$. Our study shows that the activation of GABAB receptors can also prevent apoptosis after a traumatic SCI.

Of major importance is the fact that our results also support the role of GABA as a molecule that promotes true axonal regeneration of descending neurons through the site of a complete SCI. Behavioural analyses were not performed to establish a relationship between increased regeneration and improved functional recovery after the treatments. First, because in our case, control animals usually reach the highest level of recovery when using the 


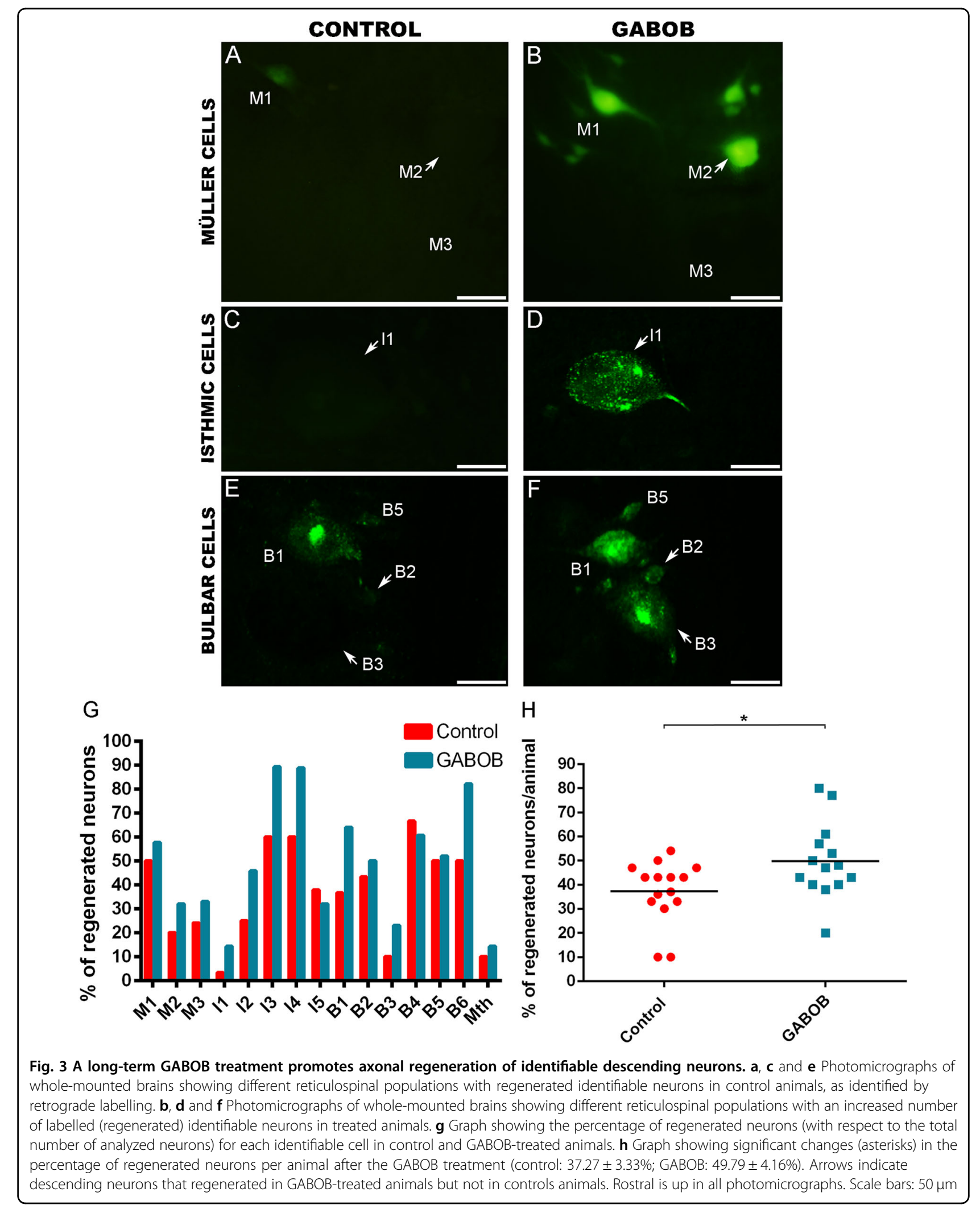




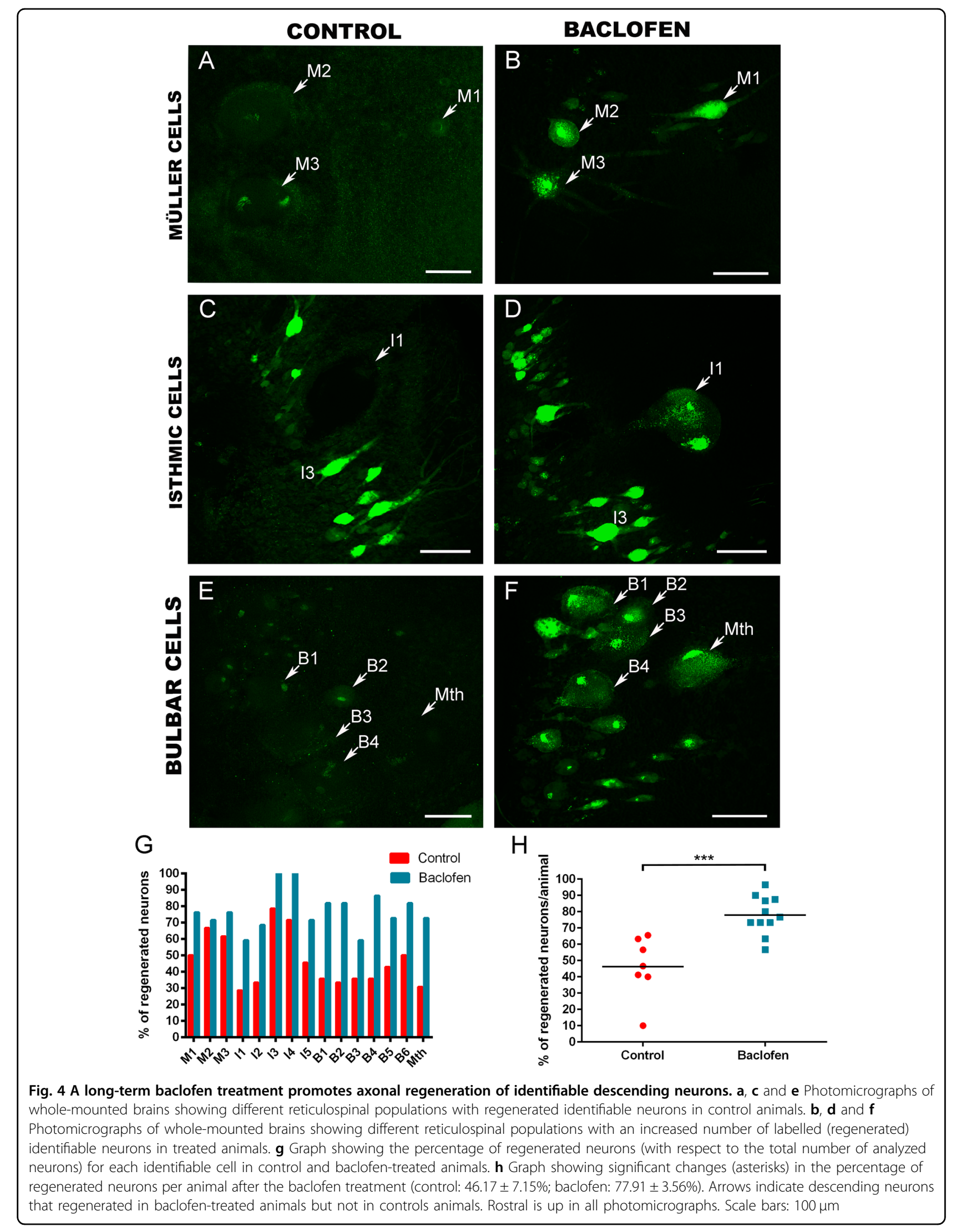




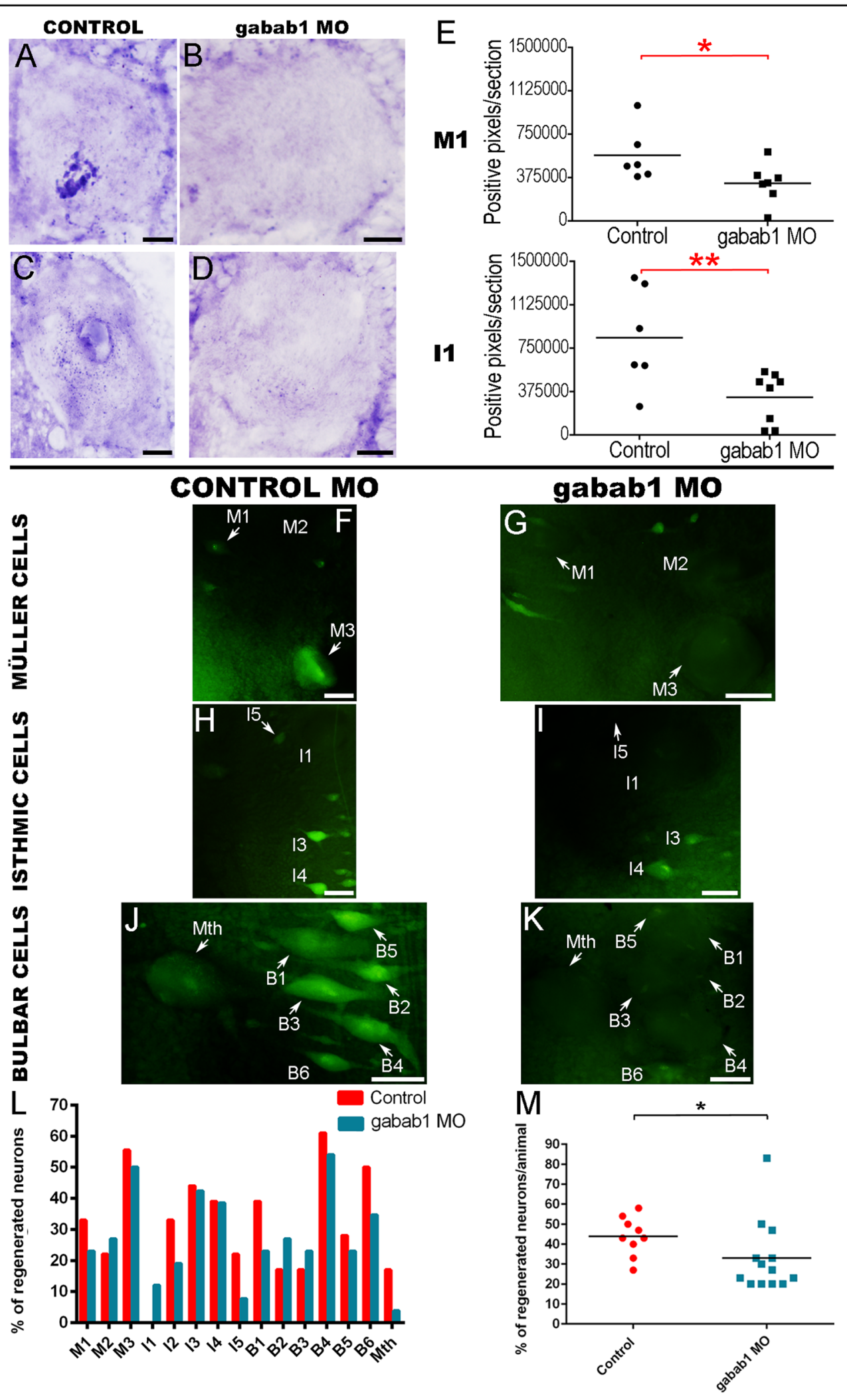

Fig. 5 (See legend on next page.) 
(see figure on previous page)

Fig. 5 Gabab1 morpholino treatments inhibit axonal regeneration of identifiable descending neurons. a, c Photomicrographs of transverse sections of $\mathrm{M} 1$ (a) and 11 (c) neurons showing the expression of the gabab1 transcript in control animals. b, d Photomicrographs of transverse sections of M1 (b) and 11 (d) neurons showing the decreased expression of gabab1 transcript in gabab1 morpholino-treated animals. e Graphs showing significant changes (asterisks) in the number of gabab1 positive pixels per section in the soma of M1 and 11 neurons after the gabab1 morpholino treatment. $\mathbf{f}, \mathbf{h}$ and $\mathbf{j}$ Photomicrographs of whole-mounted brains showing different reticulospinal populations with regenerated identifiable neurons in animals treated with the control morpholino. $\mathbf{g}, \mathbf{i}$, k: Photomicrographs of whole-mounted brains showing different reticulospinal populations with fewer regenerated identifiable neurons in animals treated with the active gabab1 morpholino. I Graph showing the percentage of regenerated neurons (respect to the total number of analyzed neurons) for each identifiable cell in control and active gabab1 morpholino-treated animals. $\mathbf{m}$ Graph showing significant changes (asterisk) in the percentage of regenerated neurons per animal after the morpholino treatment (control mismatch morpholino: $43.89 \pm 3.26 \%$; gabab1 active morpholino: $33 \pm 5 \%$ ). Arrows indicate descending neurons that regenerated in gabab1 morpholino-treated animals but not in controls animals treated with the mismatch control morpholino. Rostral is up in photomicrographs (f) to (k). Scale bars: black, $20 \mu \mathrm{m}$; white, $50 \mu \mathrm{m}$

Ayers test (see ref. ${ }^{39}$ ) and also because the treated animals were in the drugs until the day of analysis. Experiments using a gabab1 morpholino demonstrated that endogenous GABA acts as a pro-regenerative factor after SCI by activating GABAB receptors. The morpholino experiments suggest that GABA might promote regeneration by activating $\mathrm{GABAB}$ receptors expressed in the axotomized descending neurons. But, we cannot rule out the possibility that GABA could also promote the regeneration of descending neurons indirectly by inhibiting other cells expressing $\mathrm{GABAB}$ receptors, like intrinsic spinal cord neurons $^{39,42}$ that could have also taken the morpholino in our experiments. Our data agree with previous in vitro or developmental studies regarding the role of GABA and GABAB receptors in neurite outgrowth ${ }^{67}$. López-Bendito and coworkers ${ }^{67}$ showed that the GABAB antagonist CGP52432 decreases the length of the leading process in migrating inhibitory neurons in brain slice cultures of mice. Also, both GABA and baclofen stimulate retinal ganglion neurite outgrowth in Xenopus cultures, and the GABAB antagonist CGP54262 shortened the developing optic projection in vivo ${ }^{68}$. But, as far as we are aware, our results are the first in vivo demonstration showing that GABA promotes axonal regrowth after a CNS injury by activating GABAB receptors. Present and previous ${ }^{37}$ results indicate that the GABAergic system of lampreys responds successfully to a SCI to limit retrograde degeneration and promote the regeneration of descending pathways.

\section{Conclusion}

We have revealed a major role of GABA and GABAB receptors in promoting the survival and regeneration of individually identifiable descending neurons of lampreys following a complete SCI. Now, it would be of interest to decipher the underlying mechanisms behind the neuroprotective and pro-regenerative effect of GABA. Based on previous results in lampreys showing a negative effect of $\mathrm{Ca}^{2+}$ in neurite outgrowth ${ }^{35,36}$, a decrease in $\mathrm{Ca}^{2+}$ levels due to the activation of GABAB receptors could be one of the key events in the inhibition of apoptosis and activation of axonal regeneration by GABA. In future studies, it might be also interesting to analyze changes in gene expression elicited by GABA signalling and the activation of GABAB receptors to reveal new pathways involved in axonal regeneration and neuronal survival in lampreys.

The present results provide further support for the idea suggesting that the lesioned spinal cord is a "new spinal cord" $^{69}$ and the importance of understanding the changes that occur after SCI in different neurotransmitter systems in the brain and in the spinal cord above and below the site of injury. This study adds to previous work revealing anatomical $^{37-39,70,71}$ and physiological ${ }^{40,72,73}$ changes in different neurotransmitter systems above and below the lesion in recovered lampreys and highlights the importance of understanding these changes before applying neuropharmacological interventions in SCI patients. Specially, when drugs affecting neurotransmission might not only modulate locomotor circuits, but also affect the process of neuronal regeneration and recovery (e.g. serotonin inhibitors/toxins: see refs. ${ }^{72,74}$; GABOB/baclofen: present results).

Our results provide a strong basis to translate this knowledge to mammalian models of SCI for the development of new therapies for patients with SCI. A recent large observational cohort study has found that the early administration of gabapentinoids (which are administered as anticonvulsants for SCI patients) improves motor recovery following $\mathrm{SCI}^{75}$. Interestingly, baclofen is also already in use in the clinic, even for the treatment of SCI patients with spasticity ${ }^{76}$ or neuropathic pain ${ }^{77}$, which could facilitate the clinical translation of similar results in pre-clinical models of SCI.

\section{Acknowledgements}

Grant sponsors: Spanish Ministry of Economy and Competitiveness and the European Regional Development Fund 2007-2013 (Grant number: BFU201456300-P) and Xunta de Galicia (Grant number: GPC2014/030). D.R.-S. was supported by a fellowship from EMBO (Ref.: 7010) to carry out a short-term stay at the laboratory of JRM. A.B.-I. was supported by a grant from the Xunta de Galicia (Grant number: 2016-PG008) and a grant from the crowdfunding platform Precipita (FECYT; Spanish Ministry of Economy and Competitiveness; grant number 2017-CP081). The authors would like to acknowledge the following individual donors of the crowdfunding campaign in Precipita: Blanca 
Fernández, Emilio Río, Guillermo Vivar, Pablo Pérez, Jorge Férnandez, Ignacio Valiño, Pago de los Centenarios, Eva Candal, María del Pilar Balsa, Jorge Faraldo, Isabel Rodríguez-Moldes, José Manuel López, Juan José Pita, María E. Cameán, Jesús Torres, José Pumares, Verónica Rodríguez, Sara López, Tania Villares Balsa, Rocío Lizcano, José García, Ana M. Cereijo, María Pardo, Nerea Santamaría, Carolina Hernández, Jesús López and Maria Maneiro. The authors thank the staff of Ximonde Biological Station for providing lampreys used in this study, and the Microscopy Service (University of Santiago de Compostela) and Dr. Mercedes Rivas Cascallar for confocal microscope facilities and help. We also thank the Director of the Central Microscopy Facility at the Marine Biological Laboratory, Louie Kerr, for technical assistance and the Marine Biological Laboratory in Woods Hole (MA) for providing support for these experiments. This article is dedicated to the memory of José Manuel Pérez Cancela (20/11/ 1975-05/03/2018) from the Ximonde Biological Station.

\section{Author details}

'Department of Functional Biology, CIBUS, Faculty of Biology, Universidade de Santiago de Compostela, 15782 Santiago de Compostela, Spain. ${ }^{2}$ Eugene Bell Center for Regenerative Biology and Tissue Engineering; Marine Biological Laboratory, 7 MBL St., Woods Hole, MA 02543, USA. ${ }^{3}$ Present address: Department of Anatomy, Neuroscience Center, University of Helsinki, Haartmaninkatu 8, 00014 Helsinki, Finland

\section{Conflict of interest}

The authors declare that they have no conflict of interest.

\section{Publisher's note}

Springer Nature remains neutral with regard to jurisdictional claims in published maps and institutional affiliations.

Supplementary Information accompanies this paper at (https://doi.org/ 10.1038/s41419-018-0704-9).

Received: 27 March 2018 Revised: 24 April 2018 Accepted: 14 May 2018 Published online: 28 June 2018

\section{References}

1. Rovainen, C. M. Regeneration of Müller and Mauthner axons after spinal transection in larval lampreys. J. Comp. Neurol. 168, 545-554 (1976).

2. Selzer, M. E. Mechanisms of functional recovery and regeneration after spinal cord transection in larval sea lamprey. J. Physiol. 277, 395-408 (1978).

3. Wood, M. R. \& Cohen, M. J. Synaptic regeneration in identified neurons of the lamprey spinal cords. Science 206, 344-347 (1979).

4. Jacobs, A. J. et al. Recovery of neurofilament expression selectively in regenerating reticulospinal neurons. J. Neurosci. 17, 5206-5220 (1997).

5. Oliphint, P. A. et al. Regenerated synapses in lamprey spinal cord are sparse and small even after functional recovery from injury. J. Comp. Neurol. 518, 2854-2872 (2010).

6. Rodicio, M. C. \& Barreiro-lglesias, A. Las lampreas como modelo animal en estudios de regeneración tras lesión medular. Rev. Neurol. 55, 157-166 (2012).

7. Barreiro-lglesias, A. "Evorego": studying regeneration to understand evolution, the case of the serotonergic system. Brain Behav. Evol. 79, 1-3 (2012).

8. Barreiro-lglesias, A. "Bad regenerators" die after spinal cord injury: insights from lampreys. Neural Regen. Res. 10, 25-27 (2015).

9. Davis, G. R. Jr. \& McClellan, A. D. Extent and time course of restoration of descending brainstem projections in spinal cord-transected lamprey. J. Comp. Neurol. 344, 65-82 (1994).

10. Armstrong, J., Zhang, L. \& McClellan, A. D. Axonal regeneration of descending and ascending spinal projection neurons in spinal cord-transected larval lamprey. Exp. Neurol. 180, 156-166 (2003).

11. Cornide-Petronio, M. E., Ruiz, M. S., Barreiro-lglesias, A. \& Rodicio, M. C. Spontaneous regeneration of the serotonergic descending innervation in the sea lamprey after spinal cord injury. J. Neurotrauma 28, 2535-2540 (2011).

12. Busch, D. J. \& Morgan, J. R. Synuclein accumulation is associated with cellspecific neuronal death after spinal cord injury. J. Comp. Neurol. 520 , 1751-1771 (2012).
13. Fogerson, S. M. et al. Reducing synuclein accumulation improves neuronal survival after spinal cord injury. Exp. Neurol. 278, 105-115 (2016).

14. Shifman, M. I., Zhang, G. \& Selzer, M. E. Delayed death of identified reticulospinal neurons after spinal cord injury in lampreys. J. Comp. Neurol. 510, 269-282 (2008).

15. Hu, J., Zhang, G. \& Selzer, M. E. Activated caspase detection in living tissue combined with subsequent retrograde labeling, immunohistochemistry or in situ hybridization in whole-mounted lamprey brains. J. Neurosci. Methods 220, 92-98 (2013).

16. Barreiro-Iglesias, A. \& Shifman, M. I. Use of fluorochrome-labeled inhibitors of caspases to detect neuronal apoptosis in the whole-mounted lamprey brain after spinal cord injury. Enzyme Res. 2012, 835731 (2012).

17. Barreiro-Iglesias, A. \& Shifman, M. I. Detection of activated caspase-8 in injured spinal axons by using fluorochrome-labeled inhibitors of caspases (FLICA). Methods Mol. Biol. 1254, 329-339 (2015).

18. Barreiro-lglesias, A., Sobrido-Cameán, D. \& Shifman, M. I. Retrograde activation of the extrinsic apoptotic pathway in spinal-projecting neurons after a complete spinal cord injury in lampreys. Biomed. Res. Int. 2017, 5953674 (2017).

19. Demediuk, P., Daly, M. P. \& Faden, A. I. Effect of impact trauma on neurotransmitter and nonneurotransmitter amino acids in rat spinal cord. J. Neurochem. 52, 1529-1536 (1989).

20. Panter, S. S., Yum, S. W. \& Faden, A. I. Alteration in extracellular amino acids after traumatic spinal cord injury. Ann. Neurol. 27, 96-99 (1990).

21. Liu, D., Thangnipon, W. \& McAdoo, D. J. Excitatory amino acids rise to toxic levels upon impact injury to the rat spinal cord. Brain Res. 547, 344-348 (1991).

22. Liu, D., Xu, G. Y., Pan, E. \& McAdoo, D. J. Neurotoxicity of glutamate at the concentration released upon spinal cord injury. Neuroscience 93, 1383-1389 (1999).

23. Xu, G. Y., Hughes, M. G., Ye, Z., Hulsebosch, C. E. \& McAdoo, D. J. Concentrations of glutamate released following spinal cord injury kill oligodendrocytes in the spinal cord. Exp. Neurol. 187, 329-336 (2004).

24. Choi, D. W. Glutamate neurotoxicity and diseases of the nervous system Neuron 1, 623-634 (1988)

25. Ransom, R. W. \& Stec, N. L. Cooperative modulation of [3H]MK-801 binding to the N-methyl-D-aspartate receptor-ion channel complex by L-glutamate, glycine, and polyamines. J. Neurochem. 51, 830-836 (1988).

26. Schlaepfer, W. W. Calcium-induced degeneration of axoplasm in isolated segments of rat peripheral nerve. Brain Res. 69, 203-215 (1974).

27. Han, D. et al. Co-activation of GABA receptors inhibits the JNK3 apoptotic pathway via the disassembly of the GluR6-PSD95-MLK3 signaling module in cerebral ischemic-reperfusion. FEBS Lett. 582, 1298-1306 (2008).

28. Zhou, C. et al. Neuroprotection of synthase (Ser847) phosphorylation through increased neuronal nitric oxide synthase and PSD95 interaction and inhibited protein phosphatase activity in cerebral ischemia. J. Neurosci. Res. 86 2973-2983 (2008)

29. Wei, X. W. et al. Neuroprotection of co-activation of GABA receptors by preventing caspase-3 denitrosylation in KA-induced seizures. Brain Res. Bull. 88, 617-623 (2012)

30. Llorente, I. L. et al. GABA(A) receptor chloride channels are involved in the neuroprotective role of GABA following oxygen and glucose deprivation in the rat cerebral cortex but not in the hippocampus. Brain Res. 1533, 141-151 (2013).

31. Liu, L. et al. Baclofen mediates neuroprotection on hippocampal CA1 pyramidal cells through the regulation of autophagy under chronic cerebral hypoperfusion. Sci. Rep. 5, 14474 (2015).

32. Kantamneni, S. in GABAB Receptor 1st edn (ed Colombo, G.) 109-128 (Springer-Verlag, Berlin, 2016).

33. Sernagor, E., Chabrol, F., Bony, G. \& Cancedda, L. GABAergic control of neurite outgrowth and remodeling during development and adult neurogenesis: general rules and differences in diverse systems. Front. Cell. Neurosci. 4, 11 (2010).

34. Gaiarsa, J. L. \& Porcher, C. Emerging neurotrophic role of GABAB receptors in neuronal circuit development. Front. Cell. Neurosci. 7, 206 (2013).

35. Ryan, S. K. et al. Glutamate regulates neurite outgrowth of cultured descending brain neurons from larval lamprey. Dev. Neurobiol. 67, 173-188 (2007).

36. McClellan, A. D. Kovalenko, M. O, Benes, J. A. \& Schulz, D. J. Spinal cord injun induces changes in electrophysiological properties and ion channel expression of reticulospinal neurons in larval lamprey. J. Neurosci. 28, 650-659 (2008).

37. Fernández-López, B., Valle-Maroto, S. M., Barreiro-Iglesias, A. \& Rodicio, M. C. Neuronal release and successful astrocyte uptake of aminoacidergic neurotransmitters after spinal cord injury in lampreys. Glia 62, 1254-1269 (2014). 
38. Fernández-López, B., Barreiro-lglesias, A. \& Rodicio, M. C. Anatomical recovery of the spinal glutamatergic system following a complete spinal cord injury in lampreys. Sci. Rep. 6, 37786 (2016).

39. Romaus-Sanjurjo, D., Valle-Maroto, S. M., Barreiro-lglesias, A., Fernández-López, B. \& Rodicio, M. C. Anatomical recovery of the GABAergic system after a complete spinal cord injury in lampreys. Neuropharmacology 131, 389-402 (2018).

40. Svensson, E., Kim, O. \& Parker, D. Altered GABA and somatostatin modulation of proprioceptive feedback after spinal cord injury in lamprey. Neuroscience 235, 109-118 (2013).

41. Barreiro-Iglesias, A., Zhang, G., Selzer, M. E. \& Shifman, M. I. Complete spinal cord injury and brain dissection protocol for subsequent wholemount in situ hybridization in larval sea lamprey. J. Vis. Exp. 92, e51494 (2014).

42. Romaus-Sanjurjo, D., Fernández-López, B., Sobrido-Cameán, D., Barreiro-lglesias, A. \& Rodicio, M. C. Cloning of the GABA(B) receptor subunits B1 and B2 and their expression in the central nervous system of the adult sea lamprey. Front. Neuroanat. 10, 118 (2016).

43. Bussières, N. \& El Manira, A. GABA(B) receptor activation inhibits N- and P/Qtype calcium channels in cultured lamprey sensory neurons. Brain Res. 847, 175-185 (1999).

44. Bundgaard, M. Brain barrier systems in the lamprey. I. Ultrastructure and permeability of cerebral blood vessels. Brain Res. 240, 55-64 (1982).

45. Bundgaard, M. \& van Deurs, B. Brain barrier systems in the lamprey. II. Ultrastructure and permeability of the choroid plexus. Brain Res. 240, 65-75 (1982).

46. Zhang, G., Jin, L. Q., Hu, J., Rodemer, W. \& Selzer, M. E. Antisense morpholino oligonucleotides reduce neurofilament synthesis and inhibit axon regeneration in lamprey reticulospinal neurons. PLOS ONE 10, e0137670 (2015).

47. Chen, J., Laramore, C. \& Shifman, M. I. The expression of chemorepulsive guidance receptors and the regenerative abilities of spinal-projecting neurons after spinal cord injury. Neuroscience 341, 95-111 (2017).

48. Hu, J. et al. The role of RhoA in retrograde neuronal death and axon regeneration after spinal cord injury. Neurobiol. Dis. 98, 25-35 (2017).

49. Kammerer, R. A. et al. Heterodimerization of a functional GABAB receptor is mediated by parallel coiled-coil alpha-helices. Biochemistry 38, 3263-3269 (1999).

50. Sobrido-Cameán, D. \& Barreiro-lglesias, A. Role of caspase-8 and Fas in cell death after spinal cord injury. Front. Mol. Neurosci. 11, 101 (2018).

51. Berdichevsky, E., Riveros, N., Sánchez-Armáss, S. \& Orrego, F. Kainate, Nmethylaspartate and other excitatory amino acids increase calcium influx into rat brain cortex cells in vitro. Neurosci. Lett. 36, 75-80 (1983).

52. Mehta, A., Prabhakar, M., Kumar, P., Deshmukh, R. \& Sharma, P. L. Excitotoxicity: bridge to various triggers in neurodegenerative disorders. Eur. J. Pharmacol. 698, 6-18 (2013).

53. Castro-Lopes, J. M., Malcangio, M., Pan, B. H. \& Bowery, N. G. Complex changes of GABAA and GABAB receptor binding in the spinal cord dorsal horn following peripheral inflammation or neurectomy. Brain Res. 679, 289-297 (1995).

54. Drexel, M. et al. Expression of GABA receptor subunits in the hippocampus and thalamus after experimental traumatic brain injury. Neuropharmacology 88, 122-133 (2015).

55. Mowery, T. M., Sarin, R. M., Kostylev, P. V. \& Garraghty, P. E. Differences in AMPA and GABAA/B receptor subunit expression between the chronically reorganized cortex and brainstem of adult squirrel monkeys. Brain Res. 1611, 44-55 (2015).

56. Huang, L. et al. Rho-kinase inhibitor prevents acute injury against transient focal cerebral ischemia by enhancing the expression and function of GABA receptors in rats. Eur. J. Pharmacol. 797, 134-142 (2017).
57. Holmes, G., \& May, W. P. On the exact origin of the pyramidal tracts in man and other mammals. Proc. R Soc. Med. 2(Neurol Sect.), 92-100 (1909).

58. Feringa, E. R. \& Vahlsing, H. L. Labeled corticospinal neurons one year after spinal cord transection. Neurosci. Lett. 58, 283-286 (1985).

59. Fry, E. J., Stolp, H. B., Lane, M. A., Dziegielewska, K. M. \& Saunders, N. R. Regeneration of supraspinal axons after complete transection of the thoracic spinal cord in neonatal opossums (Monodelphis domestica). J. Comp. Neurol. 466, 422-444 (2003)

60. Hains, B. C., Black, J. A. \& Waxman, S. G. Primary cortical motor neurons undergo apoptosis after axotomizing spinal cord injury. J. Comp. Neurol. 462 328-341 (2003).

61. Wu, K. L., Chan, S. H., Chao, Y. M. \& Chan, J. Y. Expression of pro-inflammatory cytokine and caspase genes promotes neuronal apoptosis in pontine reticular formation after spinal cord transection. Neurobiol. Dis. 14, 19-31 (2003).

62. Lee, B. H. et al. Injury in the spinal cord may produce cell death in the brain. Brain Res. 1020, 37-44 (2004).

63. Klapka, N. et al. Suppression of fibrous scarring in spinal cord injury of rat promotes long-distance regeneration of corticospinal tract axons, rescue of primary motoneurons in somatosensory cortex and significant functional recovery. Eur. J. Neurosci. 22, 3047-3058 (2005).

64. Nielson, J. L. et al. Unexpected survival of neurons of origin of the pyramidal tract after spinal cord injury. J. Neurosci. 30, 11516-11528 (2010).

65. Nielson, J. L., Strong, M. K. \& Steward, O. A reassessment of whether cortical motor neurons die following spinal cord injury. J. Comp. Neurol. 519 2852-2869 (2011)

66. $\mathrm{Fu}, \mathrm{P} ., \mathrm{Wu}, \mathrm{Q} ., \mathrm{Hu}, \mathrm{J} ., \mathrm{Li}, \mathrm{T}$. \& Gao, F. Baclofen protects primary rat retinal ganglion cells from chemical hypoxia-Induced apoptosis through the Akt and PERK Pathways. Front. Cell. Neurosci. 10, 255 (2016).

67. López-Bendito, $\mathrm{G}$. et al. Blockade of $\mathrm{GABA}(\mathrm{B})$ receptors alters the tangential migration of cortical neurons. Cereb. Cortex 13, 932-942 (2003).

68. Ferguson, S. C. \& McFarlane, S. GABA and development of the Xenopus optic projection. J. Neurobiol. 51, 272-284 (2002).

69. Parker, D. The lesioned spinal cord is a "new" spinal cord: evidence from functional changes after spinal injury in lamprey. Front. Neural Circuits 11, 84 (2017).

70. Cohen, A. H., Abdelnabi, M., Guan, L., Ottinger, M. A. \& Chakrabarti, L. Changes in distribution of serotonin induced by spinal injury in larval lampreys: evidence from immunohistochemistry and HPLC. J. Neurotrauma 22, 172-188 (2005).

71. Fernández-López, B. et al. Full anatomical recovery of the dopaminergic system after a complete spinal cord injury in lampreys. Neural Plast. 2015, 350750 (2015).

72. Becker, M. I. \& Parker, D. Changes in functional properties and $5-H T$ modulation above and below a spinal transection in lamprey. Front. Neural Circuits 8, 148 (2015).

73. Hoffman, N. \& Parker, D. Interactive anssssd individual effects of sensory potentiation and region-specific changes in excitability after spinal cord injury. Neuroscience 199, 563-576 (2011).

74. Barreiro-lglesias, A. et al. Serotonin promotes development and regeneration of spinal motor neurons in zebrafish. Cell Rep. 13, 924-932 (2015).

75. Warner, F. M. et al. Early administration of gabapentinoids improves motor recovery after human spinal cord injury. Cell Rep. 18, 1614-1618 (2017).

76. Agarwal, S., Patel, T., Shah, N. \& Patel, B. M. Comparative study of therapeutic response to baclofen vs tolperisone in spasticity. Biomed. Pharmacother. 87, 628-635 (2017)

77. Lee, S., Zhao, X., Hatch, M., Chun, S. \& Chang, E. Central neuropathic pain in spinal cord injury. Crit. Rev. Phys. Rehabil. Med 25, 159-172 (2013). 